\title{
VIOLENCIA DE GÉNERO CONTRA NIÑAS Y ADOLESCENTES EN SANTO DOMINGO (REPÚBLICA DOMINICANA).
}

\author{
Sandra Laguna Allué \\ Educadora Social \\ Ana Isabel Isidro de Pedro \\ Profesora Titular de E.U. Departamento de Psicología Social y Antropología. Universidad de Salamanca. \\ Email: anyis@usal.es
}

https://doi.org/10.17060/ijodaep.2017.n1.v2.933

Fecha de Recepción: 9 Febrero 2017

Fecha de Admisión: 1 Abril 2017

\begin{abstract}
RESUMEN
El presente trabajo aborda el fenómeno del abuso sexual a niñas y a adolescentes en Santo Domingo (República Dominicana) mediante un estudio de campo. La muestra se obtuvo en un centro de acogida en el cual se hospedan, en régimen de internamiento temporal, menores víctimas de abusos sexuales, prostitución, explotación sexual o bien sin recursos, en desprotección y/o desamparo. Más concretamente, se buscó analizar dicho fenómeno desde la perspectiva de género mediante un estudio de casos. La información se desglosó en varias unidades de estudio, mediante un análisis de categorías inductivo. Las unidades de análisis contempladas hacen referencia al contexto social y familiar de las menores, al conocimiento de la igualdad de género que poseen las mismas, al peso de las creencias machistas, etc. Los casos estudiados presentan una sociedad estructurada en base al género y, en este sentido, también las familias -en su mayoría desestructuradas- siguen una jerarquía patriarcal muy arraigada que legitima las diferentes formas de violencia contra la mujer y no parece esclarecer entre familiares del sexo opuesto las barreras incestuosas. Además, el sentimiento de masculinidad imperante se acopla a las desigualdades de género concernientes al ámbito laboral y profesional. Esto produce un falso empoderamiento sentido por las niñas, que en una sociedad que las cosifica extraordinariamente pretenden conseguir, a través de sus cuerpos, un hombre que las proveerá, lo cual contribuye a reforzar, mantener y perpetuar estas prácticas. En este sentido, se pudo comprobar cómo el abuso sexual es una expresión del machismo. La relevancia de tal conclusión radica en cómo la gran intensidad con la que la población vive los estrictos roles de género y sus correspondientes creencias machistas van en detrimento de ambos géneros.

Palabras clave: Género, Violencia de género, Dominación masculina, Violencia contra las mujeres
\end{abstract}




\title{
VIOLENCIA DE GÉNERO CONTRA NIÑAS Y ADOLESCENTES EN SANTO DOMINGO (REPÚBLICA DOMINICANA)
}

\begin{abstract}
The present work deals with the phenomenon of sexual abuse against both child and teenage girls in Santo Domingo (Dominican Republic) through a field study. The sample was obtained from a shelter where, in a temporary internment regime, either victims of sexual abuse, prostitution, sexual exploitation or simply people with lack of resources, lack of protection and / or helplessness are hosted. Specifically, we looked for analyzing this phenomenon from a gender perspective through a case study. The information was broken down into several units of study, using an inductive category analysis. The units of analysis examined make reference to the social and family context of the minors, to the knowledge of the gender equality that they possess, to the weight of the male chauvinism beliefs, etc. The cases studied show a structured society based on gender and, in this sense, also the families -mostly unstructured-follow a strength patriarchal hierarchy that legitimizes the different forms of violence against women and does not seem to clarify the incestuous barriers between relatives of the opposite sex. In addition, the prevailing masculinity sentiment is coupled with gender inequalities concerning the labor and professional areas. This produces a false empowerment, felt by the girls who try to obtain through their bodies, in a society that treat them as an object, a supplier man, what contributes to reinforce, maintain and perpetuate these practices. In this sense, it was verified how the sexual abuse is an expression of the machismo. The relevance of such a conclusion lies in how the great intensity with which the population lives the strict gender roles and their corresponding male chauvinism beliefs is detrimental for both genders.

Keywords: Gender, Gender violence, Male domination, Violence against women
\end{abstract}

\section{ANTECEDENTES}

Según las investigaciones que nos ofrecen numerosos estudios de Latinoamérica y el Caribe, se estima que el $20 \%$ de las mujeres y entre el $5 \%$ y el $10 \%$ de los hombres sufrieron abusos sexuales durante la infancia. En concreto, el $27 \%$ de las mujeres y niñas en 2010 notificaron haber sufrido violencia sexual ocasionada por alguien que no era su pareja (Contreras, Bott, Guedes y Dartnall, 2010).

República Dominicana es un país en vías de desarrollo y cuenta con una sociedad de enraizado carácter patriarcal, donde sus estructuras sociales están organizadas en base al género (Sagot, 2008). Asimismo, la violencia sexual contra las mujeres es un fenómeno muy arraigado en dicha región, resultante de la herencia histórica que se arrastra y debido a los estrictos soportes morales que respaldan las normas jerárquicas de género, que definen los diferentes roles de mujeres y hombres (Contreras, Bott, Guedes y Dartnall, 2010). En definitiva, la situación de violencia contra las niñas y adolescentes (NA ${ }^{1}$ ) es muy variada y en ella interaccionan altos niveles de desigualdad y discriminación, pobreza y violencia social (UNICEF, 2007).

\section{METODOLOGÍA}

Con respecto a los procedimientos metodológicos para el estudio de los casos de abuso sexual infantil en Santo Domingo, la investigación se ha enmarcado en el paradigma cualitativo, puesto que el objetivo principal es conocer el fenómeno del abuso sexual infantil en profundidad para visibilizar dicho fenómeno; no establecer generalizaciones.

La metodología cualitativa produce un conocimiento científico muy rico, se construye teniendo como base el estudio de un contexto singular integrando descripciones y narraciones realizadas por el investigador, pero partiendo de las experiencias de los protagonistas (Bisquerra, 2004). En este sentido, la estrategia de investigación utilizada ha sido el estudio de casos de tipo interpretativo ya que, según Nieto y Rodríguez (2010), los estudios de caso se identifican por abordar con gran inten- 
sidad una unidad, que puede ser una persona, un grupo o bien un sistema cultural activo (Feagin, Orum y Sjoberg, 1991, citado en Álvarez y San Fabián, 2012) o bien, tienen por objetivo comprender "el significado de una experiencia" (Serrano, 1994, p. 81).

Las fases que han guiado el proceso de investigación han sido las propuestas por Montero y León (2002, p. 506): diseño del estudio de caso, elaboración de una lista de preguntas, localización de las fuentes de datos, análisis e interpretación de los datos y elaboración del informe.

Dicha investigación empírica se situó en un contexto específico (hogar de paso para menores abusadas), durante tres meses, cinco días a la semana. Respecto a los casos a estudiar, contábamos con once niñas en el centro, sus edades estaban comprendidas entre 7 y 17 años. Sin embargo, las Informantes Clave (IC) seleccionadas para la recolección de la información fueron cuatro NA con casos de abuso sexual ya que, en su mayoría, las otras menores se encontraban en el centro por motivos de Explotación Sexual Comercial y/o desamparo y no son competencia de este estudio.

No fueron las únicas fuentes de información con las que contamos ya que, durante la estancia en el país se ha estado en continua convivencia con comunitarios de diferentes sectores, de los cuales se ha procurado obtener más información complementaria acerca del tema, puesto que la intencionalidad del estudio de casos es conocer el fenómeno sin olvidar que están contextualizados en una realidad (Álvarez y San Fabián, 2012).

En cuanto a la obtención empírica de los datos, nos servimos del análisis in situ y de la observación de la realidad. Asimismo, se obtuvo información de las entrevistas que se realizaban a las niñas y a su acompañante (padres, madres, tutores, abuelos) para determinar la acogida o no en el centro.

Las técnicas de recopilación de datos utilizadas fueron fundamentalmente dos: observación participante y entrevistas. Autores como Mead, Blumer y Goffman, citados en Bisquerra (2004), establecen que las estancias largas acompañando a grupos facilitan la recolección de las interacciones que se producen entre sus miembros, es decir, datos muy ricos que de otra manera serían muy difíciles de lograr y comprender, siendo éstos recogidos en un diario de campo.

Del mismo modo, nos apoyamos en los informes clínicos de los casos de las NA. Tras conocerlos, empezamos registrando observaciones de conductas e interacciones relevantes para el estudio. Se partió de las observaciones realizadas para elaborar unos temas que servirían de guión para formular las preguntas abiertas que se efectuarían en las entrevistas, con la finalidad de recopilar narrativas, impresiones y opiniones. Se pretendía que dichos temas atendiesen a los intereses investigadores, sin llegar a ser en ningún momento entrevistas estructuradas. Los temas a tratar fueron: contexto socio-familiar y nivel educativo de las NA, contextualización de los abusos, conocimientos acerca de la igualdad de género y/o educación sexual y demandas y necesidades de las NA durante su estancia en el hogar de paso.

Para el análisis de información cualitativa hemos seleccionado el análisis de contenido mediante el uso de categorías (Álvarez-Gayou, 2005; Miles y Huberman, 1994; Rubin y Rubin, 1995, citados en Fernández Núñez, 2006). Dicha información cualitativa comprende las narrativas, los discursos y las respuestas a entrevistas no estructuradas o semi-estructuradas (preguntas abiertas). Para esto nos hemos apoyado en la "tradición sociológica" (Fernández Núñez, 2006, p. 2), puesto que incluye los modelos de decisión etnográficos.

Se ha seguido entonces un proceso de análisis de categorías inductivo (Miles y Huberman, 1994, citado en Fernández Núñez, 2006). A través de los datos obtenidos de las observaciones y las entrevistas se han desglosado los temas, codificando la información en categorías. Este proceso consiste en concentrar la información obtenida en categorías formadas por ideas y constructos establecidos por el investigador (Rubin y Rubin, 1995, citado en Fernández Núñez, 2006). 


\section{VIOLENCIA DE GÉNERO CONTRA NIÑAS Y ADOLESCENTES EN SANTO DOMINGO (REPÚBLICA DOMINICANA)}

Las categorías fueron: contexto socio-familiar de las NA, abuso sexual a NA como expresión de la masculinidad y de las desigualdades de género, conocimientos que las NA poseen sobre la equidad de género y conocimiento de las intervenciones que se han llevado a cabo en el hogar.

Para lograr lo anteriormente mencionado, se diseñó una hoja de control a partir de una serie de categorías donde registrar las variables de identificación y los aspectos más distinguidos de las Informantes Clave. Se reseñaron aspectos comunes y diferenciales que nos permitieron después crear una intervención socioeducativa adecuada e integral.

Las variables contempladas hacen referencia a:

La edad de las NA.

Estilo familiar (estructurado o desestructurado) y tipo de parentalidad.

Entorno natural de las NA y nivel socioeconómico de la familia.

Pertenencia o no del agresor al entorno cercano de la familia 0 a la misma familia.

Naturaleza (tipo de estrategia utilizada por el agresor) y tipo (abuso sexual vs. agresión sexual).

Finalmente, con el propósito de otorgarle validez y fiabilidad a la investigación, se han utilizado dos métodos que evitan falsas percepciones y contrastan la información recopilada (Yin, 1989, citado en Martínez Carazo, 2006). Por un lado, se ha utilizado la "Triangulación de Métodos" (Álvarez y San Fabián 2012, p. 9) con la finalidad de contrastar y cotejar la información obtenida a través de la observación participante con la información obtenida de las entrevistas. Por otra parte, se ha utilizado la estrategia de "Contextualización" (Álvarez y San Fabián, 2012, p. 9). La contextualización tiene una orientación etnográfica y sirve para comprender las acciones humanas dentro del contexto social y cultural en el que acontecen. Consiste en mostrar las reglas sociales que siguen las unidades de análisis para otorgar fiabilidad y validez a la investigación (Velasco y Díaz de Rada, 2006, citado en Álvarez y San Fabián, 2012).

\section{RESULTADOS}

Las conclusiones del estudio son de tipo interpretativo puesto que se ha acumulado información sobre dicho fenómeno con la intención de cotejarla con teorías ya existentes (Nieto y Rodríguez, 2010).

En primer lugar, los contextos socio familiares en los que se desarrollan las NA son de nivel socioeconómico bajo y están influenciados por una gran presencia de dinámicas violentas a nivel familiar y social que son, en sí mismos, fenómenos estructurales.

Estos tipos de violencia se materializan en conductas y en -aparentemente- inofensivas normas sociales. Éstas se transmiten de generación en generación y del hogar a la calle. Parece razonable pensar que las personas residentes en dicho contexto tendrán mayor tolerancia a la violencia y que, en tal ambiente, los entornos son violentos (Buvinic, Morrison y Shifter, 1999). Cuestión que también encontramos presente en estudios de UNICEF (2007), donde cercioran que el riesgo de sufrir violencia sexual se acentúa en entornos cuyas redes comunitarias están débiles.

En este sentido, los contextos naturales de las NA presentan familias desestructuradas, muy numerosas y, a veces, en condiciones de hacinamiento, ubicadas en entornos periféricos muy marcados por la pobreza. En las familias mencionadas, los progenitores cuentan con poca disponibilidad emocional e incurren en la dicotomía de los estilos familiares extremos: negligentes 0 autoritarios. Asimismo, en la historia familiar de las NA están muy presentes constantes abandonos y reagrupaciones con otras familias.

De igual forma, podemos también observar cuán marcados están los roles de género en las dinámicas familiares. Habitualmente la ausencia (temporal o no, con su correspondiente retorno) es de los padres. Las creencias culturales son sumamente fuertes, las niñas observan en su casa que 
sus madres no trabajan 0 , si lo hacen, son condenadas socialmente por "abandonar el cuidado de sus hijos/as y desatender la casa"; incluso ellas mismas creen que no tienen por qué trabajar cuando conviven con un hombre. En consecuencia, la responsabilidad de los varones con respecto a las tareas del hogar se limita a aportar sustento económico y se desprenden de los hijos fácilmente.

La ONE (2014), de igual manera afirmó que la incorporación de la mujer al mercado laboral va en aumento, pero que todavía es muy baja y el mismo colectivo prefiere limitarse al ámbito doméstico para cumplir con las expectativas que la sociedad pone sobre ellas. Este panorama, junto con las identidades de género tradicionales que se transmiten, crean situaciones de más vulnerabilidad para las mujeres a cualquier edad, que ya desde pequeñas son educadas para que busquen a un hombre al que deben complacer para tener recursos (UNICEF, 2007).

Otra creencia muy extendida, relacionada con lo mencionado en párrafos anteriores, es que las familias que tienen hijas tienen mucha suerte, ya que pueden "sacar a la familia de la pobreza gracias a que son féminas y pueden ganar dinero a través de su cuerpo", muchas veces son los mismos familiares los que las animan a prostituirse o negocian con la comunidad para que abusen de ellas a cambio de dinero.

Así, se puede apreciar que los abusos sexuales están despenalizados a nivel social. Las familias piden dinero a los agresores sexuales a cambio de no efectuar ninguna denuncia. El sentimiento de responsabilidad asociado al bienestar de los hijos/as es bastante preocupante.

Asimismo, los responsables de las menores que solicitaron el ingreso al Hogar no aludían como motivo de la solicitud de ingreso en el centro a la preocupación acerca del suceso de abuso y sus posibles consecuencias, sino al comportamiento "rebelde" o "insoportable" de las NA y las consecuencias del mismo (pérdida de la valía como mujer y que el hecho de ser agresiva no es adecuado para una mujer).

La presente investigación buscaba también contextualizar el abuso desde la perspectiva de género. Nuestra investigación pretendía encuadrar los abusos en dos modelos explicativos: el Modelo Multifactorial propuesto por Trepper y Barret, el cual tiene en cuenta "factores socioambientales, variables culturales, factores de la familia de origen del abusador y factores psicológicos individuales" (González, Martínez, Leyton y Bardi, 2004, p. 7). Y el Modelo de Confluencia (Malamuth et al., 1991), que considera los siguientes factores como causales o de riesgo para sufrir abusos 0 agresiones sexuales: masculinidad hostil, actitudes violentas, delincuencia y conducta antisocial, sexualidad impersonal y factores de riesgo ambientales. Parte de los datos obtenidos sí que nos permitirían basarnos en estos modelos explicativos, aunque no se recogieron datos suficientes como para hacer tales afirmaciones. Sin embargo, lo que sí hemos encontrado representado es que el género es transversal a todas las categorías existentes en las que se pueda dar el abuso pero no podemos realizar una afirmación que sitúe a las construcciones sociales como única causa de estos actos (López, 2005).

Pese a ello, sí se ha comprobado que el ejercicio de los roles tradicionales, las desigualdades económicas, la cosificación de los cuerpos y la masculinidad hostil se constituyen como factores de riesgo para el abuso sexual. De la misma forma, en comunidades tan deterioradas la violencia estructural es un componente que se materializa e influye en la tolerancia hacia los abusos sexuales, así como en los abusadores sexuales que, en su mayoría, no presentan ninguna patología como popularmente se cree, sino que se valen de las cualidades asociadas a la masculinidad, como "la fuerza, la coerción, la presión (...) para doblegar la voluntad de una mujer adulta 0 abusar de una menor, especialmente de las niñas" (López, 2005, p. 99).

Otra actitud y/o creencia encontrada está presente también en el ya mencionado estudio realizado por UNICEF (2010) como factor coadyuvante de la violencia sexual. Se trata de la creencia de 
la "genitalización incontrolable", lo que denota un fuerte machismo en el proceso de socialización; cuando en el otro extremo de esa dimensión se está asociando la sexualidad femenina únicamente a las relaciones que incluyen afecto.

Entonces, naturalizar la supuesta "genitalización incontrolable de los hombres", sería igual que minimizar la violencia masculina como algo inherente a los varones (Duarte, Gómez y Carrillo, 2011) creencia aceptada y tolerada socialmente.

En esta línea, también estaría presente el interés público acerca de lo que una mujer puede o no puede hacer y más cuando se habla de estigma social al sufrir un abuso o cuando se atribuyen las causas del abuso sexual al hecho de ser "una niña de calle" o por "seducir o provocar". Conducta que, según lo que establece la A.P.A. (2007), está producida por la sexualización de los cuerpos femeninos.

\section{CONCLUSIONES}

En Santo Domingo, las identidades genéricas tradicionales se requieren como elementos indispensables en las relaciones entre los dos grupos, en tanto que ambos buscan dichas cualidades en Ios otros y viceversa (Duarte, Gómez y Carrillo, 2011). Las mujeres entrevistadas en el estudio de UNICEF (2010), igual que en nuestra investigación, hablan acerca de la necesidad de salir del hogar de origen y explican que necesitan de los hombres para poder hacer frente a sus gastos. Los consideran sus "proveedores" de recursos económicos y esta cualidad se normaliza tanto como que las mujeres deben ubicarse en el hogar.

Se ha observado que dicho patrón se repite en la mayoría de las niñas del centro y en la sociedad dominicana en general, mostrando entonces un desconocimiento claro acerca de las implicaciones de la igualdad de género. La creencia de que los roles tradicionales vinculan a la mujer al ámbito del hogar y mantienen al hombre en un alejamiento empático de las mismas, retroalimentan la desvalorización cultural del colectivo femenino.

Podríamos concluir identificando los resultados de nuestro estudio con las ideas propuestas por Sáez, Valor-Segura y Expósito (2012), quienes exponen que la sexualización sirve de herramienta para atraer y es una "una manera de obtener las recompensas" (Fiske et al., 1991, citado en Sáez, Valor-Segura y Expósito, 2012, p. 43). En nuestro estudio, se ha comprobado que la sensación de "falso empoderamiento" está muy extendida. En este sentido Lerum y Dworkin (2009), citados en Sáez, Valor-Segura y Expósito (2012), establecen que algunas mujeres experimentan falsas sensaciones de empoderamiento a través de la cosificación, es decir, encuentran poder en ella. Sin embargo, la A.P.A., (2007) considera que asumir la cosificación como algo positivo, es un factor limitante puesto que si se abandonan los cánones de belleza que les permiten ser deseadas, volverán a estar en la esfera de la exclusión. Esto explicaría entonces que en una sociedad con un carácter patriarcal tan arraigado y con una brecha económica tan marcada entre mujeres y hombres -además de que están desvalorizadas a nivel social- el colectivo femenino trate de adquirir más poder a través de la cosificación.

En esta línea, vamos a sentar las bases de una intervención socioeducativa integral que aborde la problemática del abuso sexual. La misma también debería realizarse en la comunidad; facilitando, en primer lugar, la relativización de la cultura mediante un pensamiento crítico. Éste es el primer paso para que pueda darse la posterior modificación. Este cambio no es sencillo, porque supone prescindir de determinados valores que están muy arraigados y se debe considerar el contexto social, puesto que la confrontación puede producir lo contrario, una radicalización de las posturas (Rodríguez y Peña, 2005). Este punto de la investigación es el que deja las puertas abiertas a poder realizar una futura intervención psicosocial comunitaria en la zona de residencia de las NA junto con 
la comunidad, que tendría por objetivo que los entornos de las NA sean más pacíficos y acogedores.

La propuesta de intervención socioeducativa está basada en los programas de Save the Children (2007) que incluyen la figura del educador social en acciones como:

Incremento del valor de la educación, mediante la misma se permitirá fomentar relaciones desde la igualdad basadas en el empoderamiento de la comunidad femenina y en el pensamiento crítico hacia las implicaciones que la cultura y el género conllevan en la vida de ambos grupos.

Establecimiento de espacios dedicados al acogimiento de las NA, basados en el enfoque sistémico, considerando que ésta es un sujeto activo en su proceso y cuyos intereses deben ser aquello que guíe cualquier intervención.

Formación de los trabajadores de los hogares en dicha problemática, así como la creación de equipos multidisciplinares que promuevan la cooperación mediante redes profesionales cuyo objetivo es la búsqueda de recursos para ofrecer una respuesta integral a la víctima y a su familia.

Identificar factores y personas protectoras pertenecientes al entorno de la víctima para su fortalecimiento. Tratamiento de las dificultades de expresión y de las habilidades sociales, para poder crear entre las NA contextos de protección y apoyo mutuo.

Inclusión de la Educación Sexual en el proceso de intervención socioeducativa, puesto que el abuso constituye una intromisión en el desarrollo psicosexual, social y afectivo que requiere de actividades psicoeducativas.

Con respecto a las NA, no se ha encontrado mejor manera para definir los pensamientos que engloban esta situación que la siguiente: "su esencia no era el ser de esa manera, sino el no poder ser de otra forma” (Lorente, 2007, p. 22). Y, tras los resultados obtenidos, se podría establecer que Ios abusos sexuales a NA sí podrían considerarse una forma de violencia contra la mujer por el hecho de ser mujer. Y ello por dos motivos principales: a) si partimos del término "violencia" es porque reconocemos la existencia de conductas intolerables; b) que dicha violencia se caracterice como "de género" es debido a su carácter jerárquico de supremacía masculina vs. subordinación femenina (Marugán, 2013).

Asimismo, que las creencias culturales y de género se constituyen como un factor relevante tanto en estos sucesos como en el posterior desarrollo de las intervenciones y de las vidas de las víctimas. En este sentido, se cree que el trabajo del educador social reside aquí, en la labor de ayudar a desaprender aquellas actitudes que adquirimos como propias debido a la escasez de reflexión y que obran en detrimento de las personas que subyacen a ambos géneros (Lorente, 2007).

\section{REFERENCIAS BIBLIOGRÁFICAS}

Álvarez-Gayou, J. L. (2005). Cómo hacer investigación cualitativa. Fundamentos y metodología. México: Paidós

Álvarez, C. y San Fabián, J. L. (2012). La elección del estudio de caso en investigación educativa. Gazeta de Antropología, 28(1), 1-12. Consultado el 30 de octubre de 2015, de: http://www.ugr.es/ pwlac/G28_14Carmen_AlvarezoseLuis_SanFabian.pdf

A.P.A. (2007). Report of the A.P.A. Task Force on the Sexualization of Girls. Consultado el 26 de abril de 2016, de: http://www.apa.org/pi/women/programs/girls/report-summary.pdf

Bisquerra, R. (2004). Metodología de la investigación educativa. Madrid: La Muralla.

Buvinic, M., Morrison, A. y Shifter, M. (1999). La violencia en América Latina y el Caribe: un marco de referencia para la acción. Inter-American Development Bank. Consultado el 3 de febrero de 2016, de: https://publications.iadb.org/handle/11319/5269

Contreras, J. M., Bott, S., Guedes, A. y Dartnall, E. (2010). Violencia sexual en Latinoamérica y el 
Caribe: Análisis de datos secundarios. Iniciativa de Investigación sobre la Violencia Sexual. Consultado el 22 de abril de 2016, de: http://www.oas.org/dsp/documentos/Observatorio/violencia_sexual_la_y_caribe_2.pdf

Duarte, E., Gómez, J. y Carrillo, C. (2011). Masculinidad y Hombre maltratador ¿Pueden las creencias de hombres y mujeres propiciar violencia de género? Revista de Psicología, 19(2), 7-30. Consultado el 5 de marzo de 2016, de http://www.revistapsicologia.uchile.cl/index.php /RDP/article/viewFile/17104/17836

Feagin, J., Orum, A. y Sjoberg, G. (1991). A case for case study. Chapel Hill, University of North Carolina Press.

Fernández Núñez, L. (2006). ¿Cómo analizar datos cualitativos? Butlleti LaRecerca, 7,1-13. Consultado el 4 de mayo de 2016, de: http://www.ub.edu/ice/recerca/pdf/ficha7-cast.pdf

Fiske, S. T., Bersoff, D. N., Borgida, E., Deaux, K. y Heilman, M. E. (1991). Social science research on trial: Use of sex stereotyping research in Price Waterhouse v. Hopkins. American Psychologist, 46, 1049-1060.

López, F. (2005). Los abusos sexuales: El riesgo de ser mujer. Feminismos, 6, 91-106. Consultado el 13 de marzo de 2016, de: http://rua.ua.es/dspace/handle/10045/3183

Lorente, M. (2007). Violencia de género, educación y socialización: acciones y reacciones. Revista de Educación, 342, 19-35. Consultado el 28 de abril de 2016, de: http://www.revistaeducacion.mec.es/re342/re342_02.pdf

Malamuth, N.M., Sockloskie, R.J., Koss, M.P. y Tanaka, J.S. (1991). Characteristics of aggressors against women: testing a model using a national sample of college students. Journal of Consulting and Clinical Psychology, 59, 670-681.

Marugán, B. (2013). Violencia de género. Eunomía. Revista en Cultura de la Legalidad, (4), 226-233. Consultado el 16 de abril de 2016, de: http://eunomia.tirant.com/wp-content/uploads/2013/03/17-Eunomia4_Marugan.pdf

Miles, M. B. y Huberman, A.M. (1994). Qualitative data analysis: An expanded sourcebook. Sage: Thousand Oaks.

Montero, I. y León, 0. (2002). Clasificación y descripción de las metodologías de investigación en psicología. International Journal of Clinical and Health Psychology, 2(3), 505-510. Consultado el 5 de mayo de 2016, de: http://www.aepc.es/ijchp/articulos_pdf/ijchp-53.pdf

Nieto, S. y Rodríguez, M. J. (2010). Investigación y evaluación educativa en la sociedad del conocimiento. Salamanca: Ediciones Universidad de Salamanca.

O.N.E. (2014). Violencia contra la mujer perpetrada por la pareja o expareja en la República Dominicana: Situación actual y estado de la información. Consultado el 15 de marzo de 2016, de: http://countryoffice.unfpa.org/dominicanrepublic/drive/VIOLENCIACONTRALVIOLEN.pdf

Rodríguez, M. C. y Peña, J. V. (2005): Identidad de género y contexto escolar: Una revisión de modeIos. Revista Española de Investigaciones Sociológicas, 112, 165-194. Consultado el 13 de abril de 2016, de: http://www.redalyc.org/articulo.oa?id=99716080005

Rubin, H. J. y Rubin, I. S. (1995). Qualitative interviewing. The art of hearing data. Sage: Thousand Oaks.

Sáez, G., Valor-Segura, I. y Expósito, F. (2012). ¿Empoderamiento o subyugación de la mujer?: Experiencias de cosificación sexual interpersonal. Psychosocial Intervention, 21(1), 41-51. Consultado el 13 de abril de 2016, de: http://scielo.isciii.es/scielo.php?pid= S11325592012000100004yscript=sci_arttext

Save the Children. (2007). El abuso sexual infantil: Manual de formación para profesionales. Consultado el 4 de abril de 2016, de: https://www.savethechildren.es/sites/default/files /imce/docs/manual_abuso_sexual.pdf 
Pérez Serrano, G. (1994). Investigación cualitativa: Retos e interrogantes. Métodos. La muralla.

UNICEF (2007). Informe ¡Ni Una Más! El derecho a vivir una vida libre de violencia en América Latina y el Caribe. Consultado el 5 de marzo de 2016, de: http://www.unicef.org/lac/Ni_una_mas(1).pdf UNICEF (2010). Estudio Cualitativo sobre Violencia, Abuso y Explotación Sexual Comercial de Niños, Niñas y Adolescentes en República Dominicana: Casos Denunciados. Resultados Preliminares. Consultado el 3 de marzo de 2016, de:

http://www.unicef.org/republicadominicana/Estudio_Cualitativo_sobre_ESC_de_NNA_Rep_Domini cana.pdf

Velasco, H. y Díaz de Rada, Á. (2006). La lógica de la investigación etnográfica. Un modelo de trabajo para etnógrafos de escuela. Madrid, Trotta.

Yin, R. (1989). Case Study Research. Design and Methods, Applied Social Research Methods Series. London: Sage publications.

${ }^{1}$ Se utilizará el acrónimo NA para designar a niñas y adolescentes durante todo el escrito. 
